CRYSTALLOGRAPHIC COMMUNICATIONS

ISSN 2056-9890

Reçu le 9 mars 2015

Accepté le 31 mars 2015

Édité par A. Van der Lee, Université de Montpellier II, France

Keywords: crystal structure; $\mathrm{NaNO}_{3}+\mathrm{RbNO}_{3}$ phase diagram; bond-valence sum; chargedistribution method

CCDC reference: 1004334 Supporting information: this article has supporting information at journals.iucr.org/e

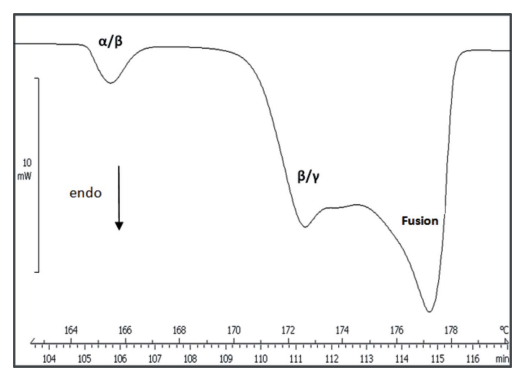

OPEN $\odot$ ACCESS

\section{Le nitrate double $\mathrm{NaRb}_{2}\left(\mathrm{NO}_{3}\right)_{3}$, composé intermédiaire du système binaire isobare $\mathrm{NaNO}_{3}+\mathrm{RbNO}_{3}$ : études thermiques et cristallographiques}

\author{
Nesrine Ksiksi, ${ }^{a}$ Mohamed Driss, ${ }^{\text {b,c }}$ Dalila Hellali, ${ }^{a}$ Abderrahmen Guesmi ${ }^{\text {b,c* } *}$ et \\ Hmida Zamali ${ }^{a}$
}

\begin{abstract}
a Université de Tunis El Manar, Faculté des Sciences, Laboratoire de Thermodynamique appliquée, El Manar II, 2092 Tunis, Tunisie, b Université de Tunis El Manar, Faculté des Sciences, Laboratoire de Matériaux et Cristallochimie, El Manar II, 2092 Tunis, Tunisie, et ' Université de Tunis El Manar, Institut Préparatoire aux Etudes d’Ingénieurs d’El Manar, El Manar II, 2092 Tunis, Tunisie. *Correspondence e-mail: abderrahmen.guesmi@ipeim.rnu.tn
\end{abstract}

Crystallographic and thermodynamic investigations of the binary $\left(\mathrm{NaNO}_{3}+\right.$ $\mathrm{RbNO}_{3}$ ) phase diagram at atmospheric pressure reveal the existence of an intermediate compound $\mathrm{NaRb}_{2}\left(\mathrm{NO}_{3}\right)_{3}$ (sodium dirubidium trinitrate) previously predicted and now reported experimentally for the first time. According to a DSC analysis, the compound exhibits three allotropic forms. In its low-temperature allotropic form ( $\alpha$ form, orthorhombic) there are two Rb ( $m$.. site symmetry) and one $\mathrm{Na}(m .$.$) independent crystallographic positions and three planar nitrate$ groups. The bond-valence-sum calculations for all atoms agree well with their oxidation states. The Rb cations are located in the (100) plane at $x=\frac{1}{2}$ with 11 oxygen coordination. The $\mathrm{Na}$ ones are in the same plane at $x=0$ and are coordinated to eight $\mathrm{O}$ atoms from six nitrate groups. The charge-distribution method has been used to evaluate the degree of distortion of the alkali polyhedra.

\section{Contexte chimique}

Les nitrates alcalins sont connus pour leurs propriétés physiques importantes. En effet, ils sont utilisés comme fondants à cause de leurs basses températures de fusion, mais ils sont utilisés également dans des électrolytes solides grâce à leurs importantes conductivités ioniques à températue ambiante (Rao et al. 2005). L'association de deux cations alcalins dans un même matériau peut induire de nouvelles propriétés, ce qui nécessite une étude préalable du diagramme de phases des nitrates correspondants. Dans ce contexte nous avons exploré le système $\mathrm{NaNO}_{3}+\mathrm{RbNO}_{3}$ où on a relevé l'existence du composé intermediaire étudié. Par ailleurs le système binaire $\mathrm{NaNO}_{3}+\mathrm{RbNO}_{3}$ a fait l'objet de plusieurs travaux antérieurs (Diogenov \& Sarapulova, 1965; Cingolani et al. 1972; Sangster, 2000). Le désaccord entre les différents travaux était au niveau de la formule du composé intermédiaire défini: Diogenov \& Sarapulova (1965) et Cingolani et al. (1972) ont proposé la formule $\mathrm{NaRb}_{2}\left(\mathrm{NO}_{3}\right)_{3}$ alors que Sangster (2000) a proposé une stoechiométrie plus riche en rubidium, soit la formule $\mathrm{NaRb}_{3}\left(\mathrm{NO}_{3}\right)_{4}$. Nos récents résultats obtenus par des études thermiques et de diffraction des rayons $\mathrm{X}$ confirment la formule exacte de ce nitrate: $\mathrm{NaRb}_{2}\left(\mathrm{NO}_{3}\right)_{3}$.

\section{Analyse thermique}

Les deux nitrates limites possèdent chacun plusieurs formes allotropiques, deux pour le nitrate de sodium et cinq pour le 


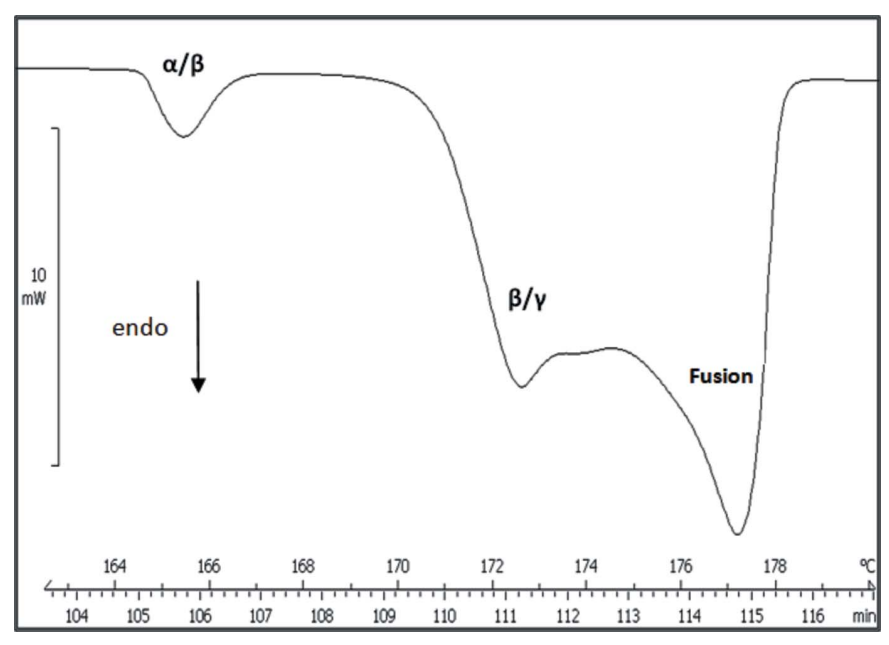

Figure 1

Courbe DSC pour $\mathrm{NaRb}_{2}\left(\mathrm{NO}_{3}\right)_{3}$ en montée de température $\left(2 \mathrm{~K} \mathrm{~min}^{-1}\right)$.

nitrate de rubidium. Afin de mettre en évidence l'existence d'éventuelles transitions de phase pour le composé étudié, nous avons réalisé une étude DSC en montée de température (Fig. 1). Nous avons déduit de ces mesures que le nitrate double étudié possède trois formes allotropiques $\alpha, \beta$ et $\gamma$ qui n'ont pas été signalées auparavant. Les températures de changement de phase sont les suivantes: $T_{\mathrm{Tr}}(\alpha \rightarrow \beta)=$ $436(1) \mathrm{K} ; T_{\text {Tr. }}(\beta \rightarrow \gamma)=442$ (1) $\mathrm{K}$ et $T_{\text {Fus. }}=451$ (1) $\mathrm{K}$.

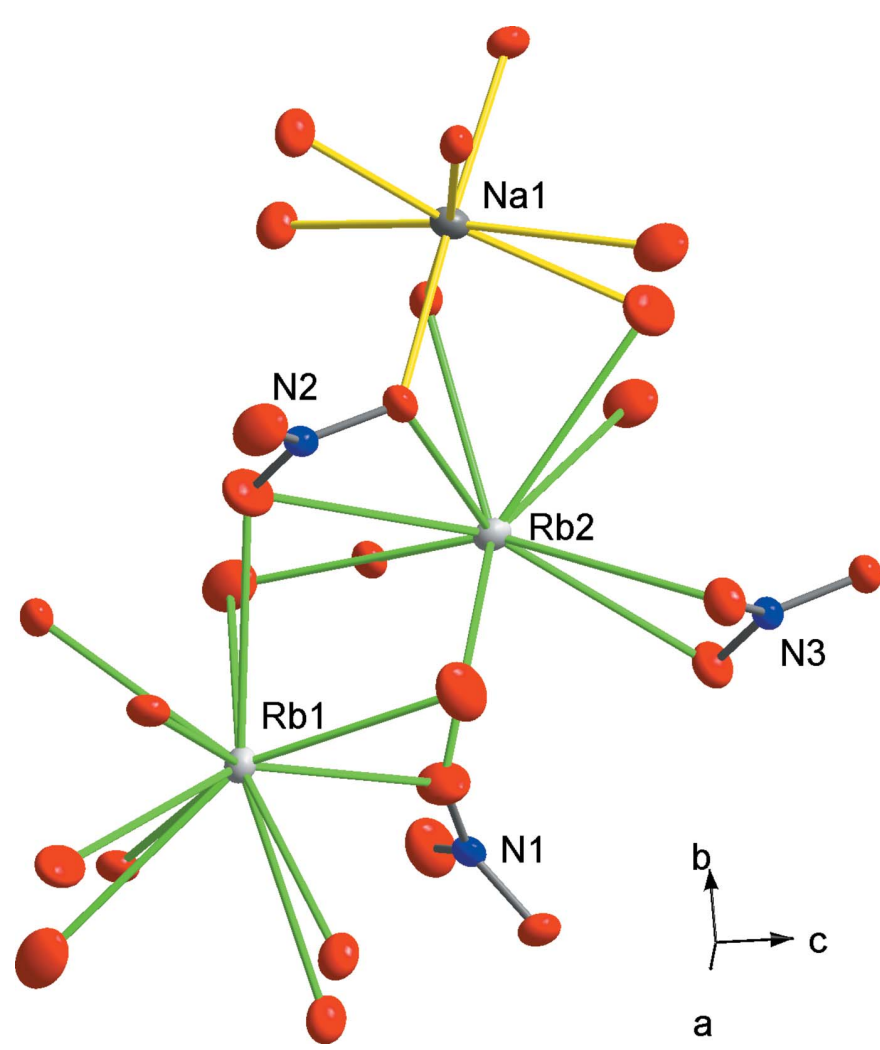

Figure 2

Modes de jonction entre les groupements nitrate et les polyèdres de coordination des cations alcalins (ellipsoïdes d'agitation thermique à $30 \%$ de probabilité de présence).

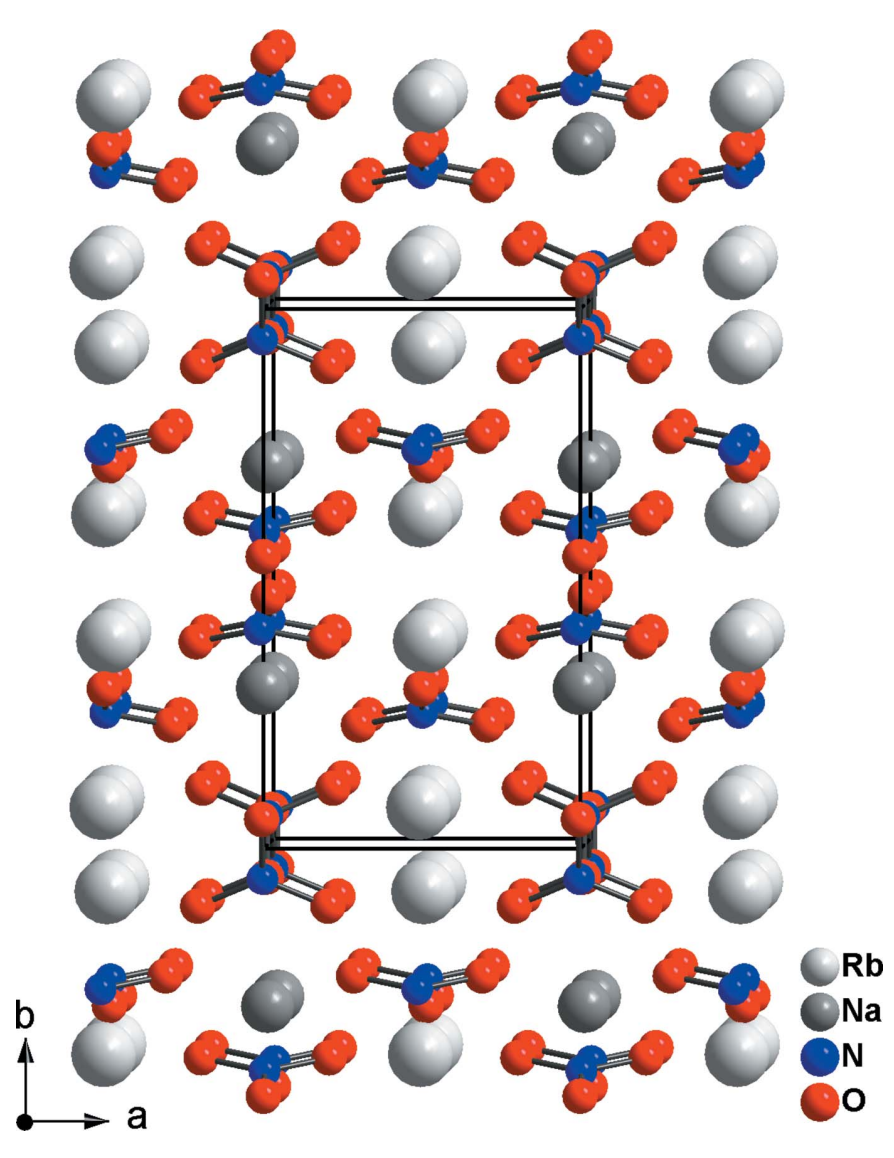

Figure 3

La structure du nitrate étudié vue selon une direction proche de [001].

\section{Commentaire structural}

La forme basse température (forme $\alpha$ ) cristallise dans le groupe d'espace $P m c 2_{1}$. Par ailleurs, l'une des formes allotropiques du composé limite au rubidium cristallise dans le groupe Pmmn (Kalliomäki \& Meisalo, 1979). L'unité asymétrique du nitrate étudié renferme un cation $\mathrm{Na}^{+}$, deux cations $\mathrm{Rb}^{+}$et trois groupements nitrate de géometrie plane (Fig. 2). Les valences des cations ainsi que celles des atomes d'oxygène sont en bon accord avec leurs degrés d'oxydation (Adams, 2004).

Les deux cations alcalins occupent des plans perpendiculaires à [100], à $x=0$ pour $\mathrm{Na}^{+}$avec une coordinence de 8 et à $x=\frac{1}{2}$ pour $\mathrm{Rb}^{+}$avec une coordinence de 11 atomes d'oxygène (Fig. 3). La grande différence entre les rayons ioniques des deux cations explique l'absence d'un désordre de substitution.

Les polyèdres de coordination des cations alcalins sont assez distordus, comme c'est généralement le cas pour ce genre de cations. La Fig. 4 montre ces polyèdres avec les distances correspondantes, les écarts entre la distance la plus longue et la plus courte pour chaque polyèdre sont $0.34 \AA$ pour Rb1, $0.38 \AA$ pour Rb2 et $0.37 \AA$ pour Na1. Pour évaluer leur distortion, nous avons examiné ces polyèdres par la méthode de distribution de charge CHARDI-IT (Nespolo, 2001; Nespolo et al. 2001). Cette méthode a montré d'une part comme la méthode BVS des 'charges' en bon accord avec les 

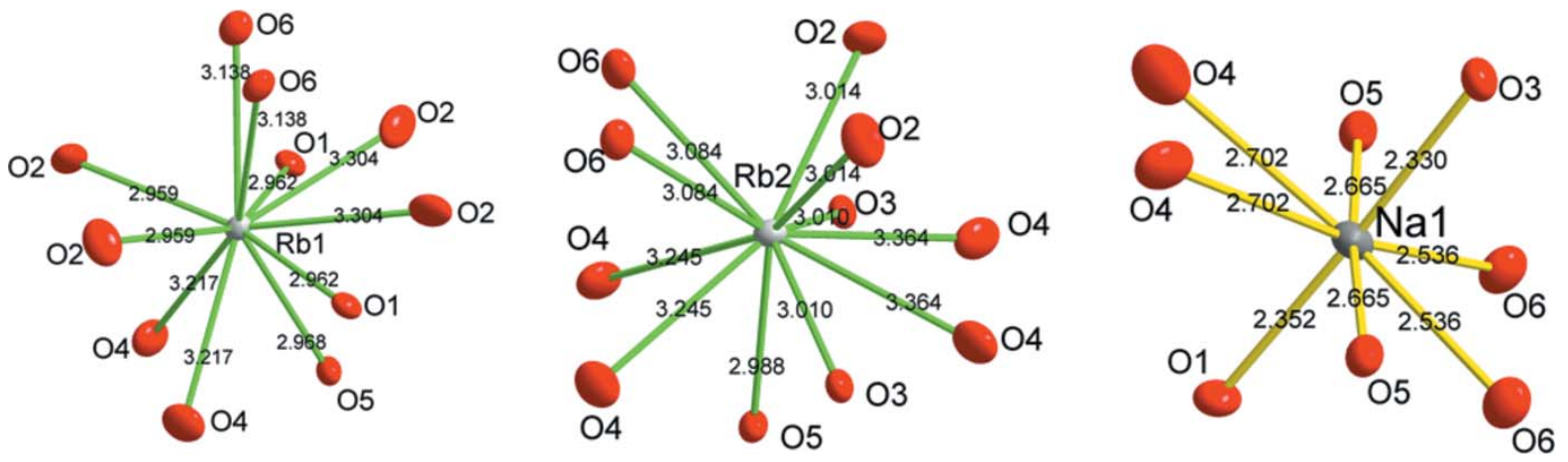

Figure 4

Les polyèdres de coordination des cations alcalins avec leurs distances correspondantes (les ellipsoïdes d'agitation thermique sont à $30 \%$ de probabilité de présence).

degrés d'oxydation de tous les atomes et d'autre part des nombres de coordination effectifs (ECoN; Hoppe, 1979) qui évaluent les degrés de distortion de ces polyèdres: plus l'ECoN s'écarte du nombre de coordination classique $\mathrm{CN}$ plus la distortion est importante; les valeurs obtenues sont les suivantes: $\mathrm{ECoN} / \mathrm{CN}(\mathrm{Rb} 1)=10.24 / 11 ; \mathrm{ECoN} / \mathrm{CN}(\mathrm{Rb} 2)=$ $10.27 / 11$ et $\mathrm{ECoN} / \mathrm{CN}(\mathrm{Na} 1)=7.03 / 8$. Les ECoNs des groupements nitrate correspondent bien à la valeur idéale $(\mathrm{CN}=3)$.

\section{Synthèse et cristallisation}

Le composé étudié a été préparé à partir d'un mélange stoechimétrique des deux nitrates alcalins correspondants. Après fusion, le mélange réactionnel a subi plusieurs cyles successifs de chauffage-refroidissement entre 298 et 473 K, température légèrement supérieure à celle de fusion du composé intermédiaire $\left[T_{\text {Fus. }}=451(1) \mathrm{K}\right]$. Après refroidissement du mélange, un fragment monocristallin pris du solide obtenu a été utilisé pour la collecte des données.

\section{Affinement}

Les données cristallographiques, les conditions de la collecte et de l'affinement sont résumées au Tableau 1. La localisation des deux cations alcalins a été basée sur leurs densités électroniques différentes ainsi que sur leurs distances par rapport aux atomes d'oxygène. Leurs taux d'occupation n'ont pas dévié de l'unité, excluant ainsi la possibilité d'existence d'un désordre de substitution. Le résidu électronique final, de 0.59 e $\AA^{-3}$, se situe à $0.93 \AA$ de Rb1. Malgré un nombre faible de paires de Friedel, mais grace à la présence de diffuseurs anomales la configuration absolue comme présentée ici semble être la bonne.

\section{Acknowledgements}

Les auteurs remercient Professeur Ahmed Driss du Laboratoire de Matériaux et Cristallochimie de la Faculté des Sciences de Tunis pour la collecte des données cristallographiques.

\section{Références}

Adams, S. (2004). SoftBV. Université Nationale de Singapour.

Brandenburg, K. (2006). DIAMOND. Crystal Impact GbR, Bonn, Allemagne.

Cingolani, A., Berchesi, M. A., Piantoni, G. \& Lecresi, D. (1972). Z. Naturforsch. Teil A, 27, 159-161.

Diogenov, G. G. \& Sarapulova, K. F. (1965). Russ. J. Inorg. Chem. 10, 1052-1054.

Duisenberg, A. J. M. (1992). J. Appl. Cryst. 25, 92-96.

Farrugia, L. J. (2012). J. Appl. Cryst. 45, 849-854.

Flack, H. D. (1983). Acta Cryst. A39, 876-881.

Harms, K. \& Wocadlo, S. (1995). XCAD4. Université de Marburg, Allemagne.

Tableau 1

Détails expérimentaux.

Données crystallines

Formule chimique

$M_{\mathrm{r}}$

Système cristallin, groupe d'espace

Température (K)

$a, b, c(\AA)$

$V\left(\AA^{3}\right)$

$Z$

Type de rayonnement

$\mu\left(\mathrm{mm}^{-1}\right)$

Taille des cristaux (mm)

Collection de données

Diffractomètre

Correction d'absorption

$T_{\min }, T_{\max }$

Nombre de réflexions mesurées,

indépendantes et observées

$[I>2 \sigma(I)]$

$R_{\text {int }}$

$(\sin \theta / \lambda)_{\max }\left(\AA^{-1}\right)$

Affinement

$R\left[F^{2}>2 \sigma\left(F^{2}\right)\right], w R\left(F^{2}\right), S$

Nombre de réflexions

Nombre de paramètres

Nombre de restraints

$\Delta \rho_{\max }, \Delta \rho_{\min }\left(\mathrm{e}^{-3}\right)$

Absolute structure

Paramètre de structure absolue

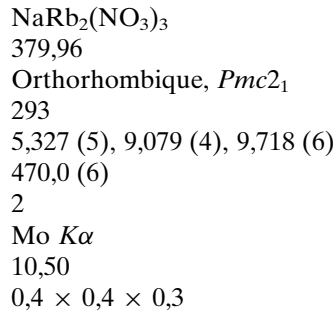

$\mathrm{NaRb}_{2}\left(\mathrm{NO}_{3}\right)_{3}$

379,96

Orthorhombique, $P m c 2_{1}$ 293

5,327 (5), 9,079 (4), 9,718 (6)

$470,0(6)$

2

Mo $K \alpha$

10,50

$0,4 \times 0,4 \times 0,3$

Enraf-Nonius CAD-4

$\psi$ scan (North et al., 1968)

$0,545,0,995$

$1474,727,662$

0,038

0,660

Programmes informatiques: CAD-4 EXPRESS (Duisenberg, 1992; Macícek \& Yordanov, 1992), XCAD4 (Harms \& Wocadlo, 1995), SHELXS97 et SHELXL97 (Sheldrick, 2008), DIAMOND (Brandenburg, 2006), WinGX (Farrugia, 2012) et publCIF (Westrip, 2010). 
Hoppe, R. (1979). Z. Kristallogr. 150, 23-52.

Kalliomäki, M. S. \& Meisalo, V. P. J. (1979). Acta Cryst. B35, 2829 2835.

Macíček, J. \& Yordanov, A. (1992). J. Appl. Cryst. 25, 73-80.

Nespolo, M. (2001). CHARDI-IT. Laboratoire CRM2, Université de Nancy I, France.

Nespolo, M., Ferraris, G., Ivaldi, G. \& Hoppe, R. (2001). Acta Cryst. B57, 652-664.
North, A. C. T., Phillips, D. C. \& Mathews, F. S. (1968). Acta Cryst. A24, 351-359.

Rao, M. M., Reddy, S. N., Chary, A. S. \& Shahi, K. (2005). Phys. B: Condens. Matter, 364, 306-310.

Sangster, J. (2000). J. Phase Equilib. 21, 241-268.

Sheldrick, G. M. (2008). Acta Cryst. A64, 112-122.

Westrip, S. P. (2010). J. Appl. Cryst. 43, 920-925. 


\section{supporting information}

Acta Cryst. (2015). E71, 455-458 [https://doi.org/10.1107/S2056989015006532]

Le nitrate double $\mathrm{NaRb}_{2}\left(\mathrm{NO}_{3}\right)_{3}$, composé intermédiaire du système binaire isobare $\mathrm{NaNO}_{3}+\mathrm{RbNO}_{3}$ : études thermiques et cristallographiques

Nesrine Ksiksi, Mohamed Driss, Dalila Hellali, Abderrahmen Guesmi et Hmida Zamali

Computing details

Data collection: CAD-4 EXPRESS (Duisenberg, 1992; Macíček \& Yordanov, 1992); cell refinement: CAD-4 EXPRESS

(Duisenberg, 1992; Macíček \& Yordanov, 1992); data reduction: XCAD4 (Harms \& Wocadlo, 1995); program(s) used to solve structure: SHELXS97 (Sheldrick, 2008); program(s) used to refine structure: SHELXL97 (Sheldrick, 2008); molecular graphics: DIAMOND (Brandenburg, 2006); software used to prepare material for publication: Win GX (Farrugia, 2012) and publCIF (Westrip, 2010).

Sodium dirubidium trinitrate

Crystal data

$\mathrm{NaRb}_{2}\left(\mathrm{NO}_{3}\right)_{3}$

$M_{r}=379.96$

Orthorhombic, $P m c 2_{1}$

Hall symbol: P 2c -2

$a=5.327(5) \AA$

$b=9.079(4) \AA$

$c=9.718(6) \AA$

$V=470.0(6) \AA^{3}$

$Z=2$

Data collection

Enraf-Nonius CAD-4 diffractometer

Graphite monochromator $\omega / 2 \theta$ scans

Absorption correction: $\psi$ scan (North et al., 1968)

$T_{\min }=0.545, T_{\max }=0.995$

1474 measured reflections

727 independent reflections

\section{Refinement}

Refinement on $F^{2}$

Least-squares matrix: full

$R\left[F^{2}>2 \sigma\left(F^{2}\right)\right]=0.024$

$w R\left(F^{2}\right)=0.062$

$S=0.83$

727 reflections

83 parameters

1 restraint
$F(000)=356$

$D_{\mathrm{x}}=2.685 \mathrm{Mg} \mathrm{m}^{-3}$

Mo $K \alpha$ radiation, $\lambda=0.71073 \AA$

Cell parameters from 25 reflections

$\theta=10-15^{\circ}$

$\mu=10.50 \mathrm{~mm}^{-1}$

$T=293 \mathrm{~K}$

Parallelepiped, white

$0.4 \times 0.4 \times 0.3 \mathrm{~mm}$

662 reflections with $I>2 \sigma(I)$

$R_{\text {int }}=0.038$

$\theta_{\max }=28.0^{\circ}, \theta_{\min }=2.2^{\circ}$

$h=-7 \rightarrow 7$

$k=-1 \rightarrow 11$

$l=-1 \rightarrow 12$

2 standard reflections every 120 reflections intensity decay: $4 \%$

$w=1 /\left[\sigma^{2}\left(F_{\mathrm{o}}^{2}\right)+(0.0532 P)^{2}\right]$

where $P=\left(F_{\mathrm{o}}^{2}+2 F_{\mathrm{c}}^{2}\right) / 3$

$(\Delta / \sigma)_{\max }<0.001$

$\Delta \rho_{\max }=0.59$ e $\AA^{-3}$

$\Delta \rho_{\min }=-0.56$ e $\AA^{-3}$

Extinction correction: (SHELXL97; Sheldrick, 2008)

Extinction coefficient: $0.036(3)$ 
Absolute structure: Flack (1983), 63 paires de Friedel

Special details

Geometry. All e.s.d.'s (except the e.s.d. in the dihedral angle between two 1.s. planes) are estimated using the full covariance matrix. The cell e.s.d.'s are taken into account individually in the estimation of e.s.d.'s in distances, angles and torsion angles; correlations between e.s.d.'s in cell parameters are only used when they are defined by crystal symmetry. An approximate (isotropic) treatment of cell e.s.d.'s is used for estimating e.s.d.'s involving 1.s. planes.

Fractional atomic coordinates and isotropic or equivalent isotropic displacement parameters $\left(\AA^{2}\right)$

\begin{tabular}{lllll}
\hline & $x$ & $y$ & $z$ & $U_{\text {iso }} * / U_{\text {eq }}$ \\
\hline $\mathrm{Rb} 1$ & 0.5 & $0.08278(8)$ & $0.33461(7)$ & $0.0293(2)$ \\
$\mathrm{Rb} 2$ & 0.5 & $0.37837(8)$ & $0.66067(7)$ & $0.0312(2)$ \\
$\mathrm{Na} 1$ & 0 & $0.7007(3)$ & $0.6151(3)$ & $0.0329(8)$ \\
$\mathrm{N} 1$ & 1 & $0.0537(7)$ & $0.6267(7)$ & $0.0292(15)$ \\
$\mathrm{O} 1$ & 1 & $-0.0580(6)$ & $0.7033(7)$ & $0.0382(14)$ \\
$\mathrm{O} 2$ & $0.7945(12)$ & $0.1047(6)$ & $0.5920(7)$ & $0.0665(17)$ \\
$\mathrm{N} 2$ & 0 & $0.4139(7)$ & $0.4141(8)$ & $0.0263(14)$ \\
$\mathrm{O} 3$ & 0 & $0.4581(7)$ & $0.5370(6)$ & $0.0374(14)$ \\
$\mathrm{O} 4$ & $0.2020(10)$ & $0.3901(5)$ & $0.3557(7)$ & $0.0615(16)$ \\
$\mathrm{N} 3$ & 0.5 & $0.2516(6)$ & $0.9835(7)$ & $0.0268(13)$ \\
$\mathrm{O} 5$ & 0.5 & $0.2981(6)$ & $1.1048(6)$ & $0.0391(14)$ \\
$\mathrm{O} 6$ & $0.7029(8)$ & $0.2278(5)$ & $0.9225(5)$ & $0.0443(11)$ \\
\hline
\end{tabular}

Atomic displacement parameters $\left(\AA^{2}\right)$

\begin{tabular}{lllllll}
\hline & $U^{11}$ & $U^{22}$ & $U^{33}$ & $U^{12}$ & $U^{13}$ & $U^{23}$ \\
\hline $\mathrm{Rb} 1$ & $0.0286(4)$ & $0.0330(4)$ & $0.0263(4)$ & 0 & 0 & $0.0045(3)$ \\
$\mathrm{Rb} 2$ & $0.0313(4)$ & $0.0257(3)$ & $0.0366(4)$ & 0 & 0 & $0.0044(3)$ \\
$\mathrm{Na} 1$ & $0.0323(15)$ & $0.0263(15)$ & $0.0400(19)$ & 0 & 0 & $-0.0054(13)$ \\
$\mathrm{N} 1$ & $0.037(3)$ & $0.022(3)$ & $0.029(4)$ & 0 & 0 & $-0.006(3)$ \\
$\mathrm{O} 1$ & $0.045(3)$ & $0.024(2)$ & $0.046(4)$ & 0 & 0 & $0.005(2)$ \\
$\mathrm{O} 2$ & $0.066(3)$ & $0.067(3)$ & $0.067(4)$ & $0.032(3)$ & $-0.027(3)$ & $-0.006(3)$ \\
$\mathrm{N} 2$ & $0.027(3)$ & $0.023(3)$ & $0.030(4)$ & 0 & 0 & $-0.002(3)$ \\
$\mathrm{O} 3$ & $0.050(4)$ & $0.033(3)$ & $0.029(3)$ & 0 & 0 & $-0.006(2)$ \\
$\mathrm{O} 4$ & $0.053(3)$ & $0.062(3)$ & $0.069(4)$ & $0.007(2)$ & $0.032(3)$ & $0.000(3)$ \\
$\mathrm{N} 3$ & $0.029(3)$ & $0.027(3)$ & $0.025(3)$ & 0 & 0 & $-0.002(3)$ \\
$\mathrm{O} 5$ & $0.057(4)$ & $0.033(3)$ & $0.027(3)$ & 0 & 0 & $-0.002(3)$ \\
$\mathrm{O} 6$ & $0.033(2)$ & $0.059(3)$ & $0.041(3)$ & $0.003(2)$ & $0.007(2)$ & $-0.007(2)$ \\
\hline
\end{tabular}

Geometric parameters $(\AA, \circ)$

\begin{tabular}{llll}
\hline $\mathrm{Rb} 1-\mathrm{O} 2$ & $2.959(6)$ & $\mathrm{Na} 1-\mathrm{N} 3^{\mathrm{xii}}$ & $2.986(4)$ \\
$\mathrm{Rb} 1-\mathrm{O} 2^{\mathrm{i}}$ & $2.959(6)$ & $\mathrm{Na} 1-\mathrm{N} 3^{\mathrm{vi}}$ & $2.986(4)$ \\
$\mathrm{Rb} 1-\mathrm{O} 1^{\mathrm{ii}}$ & $2.962(4)$ & $\mathrm{Na} 1-\mathrm{Rb} 1^{\text {iii }}$ & $3.938(3)$ \\
$\mathrm{Rb} 1-\mathrm{O} 1^{\mathrm{iii}}$ & $2.962(4)$ & $\mathrm{Na} 1-\mathrm{Rb} 1^{\text {1iii }}$ & $3.938(3)$ \\
$\mathrm{Rb} 1-\mathrm{O} 5^{\mathrm{iv}}$ & $2.968(6)$ & $\mathrm{N} 1-\mathrm{O} 2$ & $1.235(6)$ \\
$\mathrm{Rb} 1-\mathrm{O}^{\mathrm{ii}}$ & $3.138(5)$ & $\mathrm{N} 1-\mathrm{O} 2^{\mathrm{xiv}}$ & $1.235(6)$
\end{tabular}




\begin{tabular}{|c|c|c|c|}
\hline $\mathrm{Rb} 1-\mathrm{O}^{\mathrm{v}}$ & $3.138(5)$ & $\mathrm{N} 1-\mathrm{O} 1$ & $1.258(9)$ \\
\hline $\mathrm{Rb} 1-\mathrm{O} 4^{\mathrm{i}}$ & $3.217(5)$ & $\mathrm{N} 1-\mathrm{Rb} 1^{\mathrm{xv}}$ & $3.565(5)$ \\
\hline $\mathrm{Rb} 1-\mathrm{O} 4$ & $3.217(5)$ & $\mathrm{N} 1-\mathrm{Rb} 1^{\mathrm{xvi}}$ & $3.565(5)$ \\
\hline $\mathrm{Rb} 1-\mathrm{O} 2^{\mathrm{ii}}$ & $3.304(7)$ & $\mathrm{O} 1-\mathrm{Na} 1^{\mathrm{xvii}}$ & $2.352(6)$ \\
\hline $\mathrm{Rb} 1-\mathrm{O} 2^{\mathrm{v}}$ & $3.304(7)$ & $\mathrm{O} 1-\mathrm{Rb}^{\mathrm{xv}}$ & $2.962(4)$ \\
\hline $\mathrm{Rb} 1-\mathrm{N} 3^{\mathrm{ii}}$ & $3.363(6)$ & $\mathrm{O} 1-\mathrm{Rb} 1^{\mathrm{xvi}}$ & $2.962(4)$ \\
\hline $\mathrm{Rb} 2-\mathrm{O}^{\mathrm{vi}}$ & $2.988(6)$ & $\mathrm{O} 2-\mathrm{Rb} 1^{\mathrm{xvi}}$ & $3.304(7)$ \\
\hline $\mathrm{Rb} 2-\mathrm{O}^{\mathrm{vii}}$ & $3.010(4)$ & $\mathrm{N} 2-\mathrm{O} 4^{\mathrm{x} v i i i}$ & $1.236(6)$ \\
\hline $\mathrm{Rb} 2-\mathrm{O} 3$ & $3.010(4)$ & $\mathrm{N} 2-\mathrm{O} 4$ & $1.236(6)$ \\
\hline $\mathrm{Rb} 2-\mathrm{O} 2^{\mathrm{i}}$ & $3.014(5)$ & $\mathrm{N} 2-\mathrm{O} 3$ & $1.259(10)$ \\
\hline $\mathrm{Rb} 2-\mathrm{O} 2$ & $3.014(5)$ & $\mathrm{N} 2-\mathrm{Rb} 2^{\mathrm{xix}}$ & 3.597 (5) \\
\hline $\mathrm{Rb} 2-\mathrm{O} 6$ & $3.084(5)$ & $\mathrm{O} 3-\mathrm{Rb} 2^{\mathrm{xix}}$ & 3.010 (4) \\
\hline $\mathrm{Rb} 2-\mathrm{O}^{\mathrm{i}}$ & $3.084(5)$ & $\mathrm{O} 4-\mathrm{Na} 1^{\mathrm{xii}}$ & $2.702(7)$ \\
\hline $\mathrm{Rb} 2-\mathrm{O} 4^{\text {viii }}$ & $3.245(5)$ & $\mathrm{O} 4-\mathrm{Rb} 2^{\mathrm{vi}}$ & $3.245(5)$ \\
\hline $\mathrm{Rb} 2-\mathrm{O} 4^{\mathrm{ix}}$ & $3.245(5)$ & $\mathrm{N} 3-\mathrm{O}^{\mathrm{i}}$ & $1.252(5)$ \\
\hline $\mathrm{Rb} 2-\mathrm{N} 3$ & $3.341(7)$ & N3-O6 & $1.252(5)$ \\
\hline $\mathrm{Rb} 2-\mathrm{O} 4^{\mathrm{i}}$ & $3.364(7)$ & $\mathrm{N} 3-\mathrm{O} 5$ & $1.252(10)$ \\
\hline $\mathrm{Rb} 2-\mathrm{O} 4$ & $3.364(7)$ & $\mathrm{N} 3-\mathrm{Na} 1^{\text {viii }}$ & $2.986(4)$ \\
\hline $\mathrm{Na} 1-\mathrm{O} 3$ & $2.330(6)$ & $\mathrm{N} 3-\mathrm{Na} 1^{\text {xiii }}$ & $2.986(4)$ \\
\hline $\mathrm{Na} 1-O 1^{x}$ & $2.352(6)$ & $\mathrm{N} 3-\mathrm{Rb}^{\mathrm{xvi}}$ & $3.363(6)$ \\
\hline $\mathrm{Na} 1-\mathrm{O}^{\mathrm{xi}}$ & $2.536(5)$ & $\mathrm{O} 5-\mathrm{Na} 1^{\text {viii }}$ & $2.665(3)$ \\
\hline $\mathrm{Na} 1-\mathrm{O}^{\mathrm{vi}}$ & $2.536(5)$ & $\mathrm{O} 5-\mathrm{Na} 1^{\text {xiii }}$ & $2.665(3)$ \\
\hline $\mathrm{Na} 1-\mathrm{O}^{\mathrm{xii}}$ & $2.665(3)$ & $\mathrm{O} 5-\mathrm{Rb} 1^{\mathrm{xx}}$ & $2.968(6)$ \\
\hline $\mathrm{Na} 1-\mathrm{O}^{\mathrm{vi}}$ & $2.665(3)$ & $\mathrm{O} 5-\mathrm{Rb} 2^{\text {viii }}$ & $2.988(6)$ \\
\hline $\mathrm{Na} 1-\mathrm{O} 4^{\mathrm{ix}}$ & $2.702(7)$ & $\mathrm{O} 6-\mathrm{Na} 1^{\text {viii }}$ & $2.536(5)$ \\
\hline $\mathrm{Na} 1-\mathrm{O} 4^{\mathrm{xiii}}$ & $2.702(7)$ & $\mathrm{O} 6-\mathrm{Rb}^{\mathrm{xvi}}$ & $3.138(5)$ \\
\hline $\mathrm{O} 2-\mathrm{Rb} 1-\mathrm{O} 2^{\mathrm{i}}$ & $64.0(3)$ & $\mathrm{O} 3-\mathrm{Na} 1-\mathrm{O} 5^{\mathrm{vi}}$ & 89.52 (14) \\
\hline $\mathrm{O} 2-\mathrm{Rb} 1-\mathrm{O} 1^{\mathrm{ii}}$ & $147.78(18)$ & $\mathrm{O} 1^{\mathrm{x}}-\mathrm{Na} 1-\mathrm{O}^{\mathrm{vi}}$ & $90.56(14)$ \\
\hline $\mathrm{O} 2^{\mathrm{i}}-\mathrm{Rb} 1-\mathrm{O} 1^{\mathrm{ii}}$ & 83.83 (19) & $\mathrm{O} 6^{\mathrm{xi}}-\mathrm{Na} 1-\mathrm{O} 5^{\mathrm{vi}}$ & $126.5(2)$ \\
\hline $\mathrm{O} 2-\mathrm{Rb} 1-\mathrm{O} 1^{\mathrm{iii}}$ & $83.83(19)$ & $\mathrm{O} 6^{\mathrm{vi}}-\mathrm{Na} 1-\mathrm{O} 5^{\mathrm{vi}}$ & 49.27 (16) \\
\hline 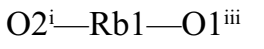 & $147.78(18)$ & $\mathrm{O} 5^{\mathrm{xi}}-\mathrm{Na} 1-\mathrm{O} 5^{\mathrm{vi}}$ & $175.6(3)$ \\
\hline 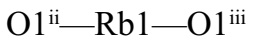 & $128.1(2)$ & $\mathrm{O} 3-\mathrm{Na} 1-\mathrm{O} 4^{\mathrm{ix}}$ & $89.6(2)$ \\
\hline $\mathrm{O} 2-\mathrm{Rb} 1-\mathrm{O} 5^{\mathrm{iv}}$ & $126.29(15)$ & $\mathrm{O} 1^{\mathrm{x}}-\mathrm{Na} 1-\mathrm{O} 4^{\mathrm{ix}}$ & $88.2(2)$ \\
\hline $\mathrm{O} 2^{\mathrm{i}}-\mathrm{Rb} 1-\mathrm{O}^{5 \mathrm{iv}}$ & $126.29(15)$ & $\mathrm{O} 6^{\mathrm{xi}}-\mathrm{Na} 1-\mathrm{O} 4^{\mathrm{ix}}$ & 164.85 (17) \\
\hline $\mathrm{O} 1^{\mathrm{ii}}-\mathrm{Rb} 1-\mathrm{O} 5^{\mathrm{iv}}$ & $74.08(13)$ & $\mathrm{O}^{\mathrm{vi}}-\mathrm{Na} 1-\mathrm{O} 4^{\mathrm{ix}}$ & $117.92(15)$ \\
\hline 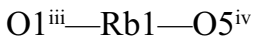 & 74.08 (13) & $\mathrm{O} 5^{\mathrm{xii}}-\mathrm{Na} 1-\mathrm{O} 4^{\mathrm{ix}}$ & $115.6(2)$ \\
\hline $\mathrm{O} 2-\mathrm{Rb} 1-\mathrm{O}^{\mathrm{ii}}$ & 90.74 (14) & 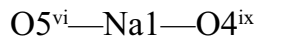 & $68.66(17)$ \\
\hline $\mathrm{O} 2^{\mathrm{i}}-\mathrm{Rb} 1-\mathrm{O} 6^{\mathrm{ii}}$ & 69.38 (13) & $\mathrm{O} 3-\mathrm{Na} 1-\mathrm{O} 4^{\mathrm{xiii}}$ & $89.6(2)$ \\
\hline 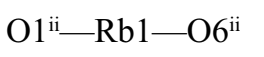 & $74.89(14)$ & $\mathrm{O} 1^{x}-\mathrm{Na} 1-\mathrm{O} 4^{\mathrm{xiii}}$ & $88.2(2)$ \\
\hline 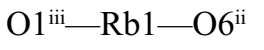 & $111.03(13)$ & $\mathrm{O} 6^{\mathrm{xi}}-\mathrm{Na} 1-\mathrm{O} 4^{\mathrm{xiii}}$ & $117.92(15)$ \\
\hline $\mathrm{O} 5^{\mathrm{iv}}-\mathrm{Rb} 1-\mathrm{O} 6^{\mathrm{ii}}$ & $142.79(13)$ & $\mathrm{O} 6^{\mathrm{vi}}-\mathrm{Na} 1-\mathrm{O} 4^{\mathrm{xii}}$ & $164.85(17)$ \\
\hline $\mathrm{O} 2-\mathrm{Rb} 1-\mathrm{O}^{\mathrm{v}}$ & $69.38(13)$ & $\mathrm{O} 5^{\mathrm{xii}}-\mathrm{Na} 1-\mathrm{O} 4^{\mathrm{xiii}}$ & $68.66(17)$ \\
\hline $\mathrm{O} 2^{\mathrm{i}}-\mathrm{Rb} 1-\mathrm{O}^{\mathrm{v}}$ & $90.74(14)$ & 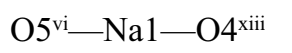 & $115.6(2)$ \\
\hline $\mathrm{O} 1^{\mathrm{ii}}-\mathrm{Rb} 1-\mathrm{O}^{\mathrm{v}}$ & $111.03(13)$ & $\mathrm{O} 4^{\mathrm{ix}}-\mathrm{Na} 1-\mathrm{O} 4^{\mathrm{xiii}}$ & $46.9(2)$ \\
\hline $\mathrm{O}{ }^{\mathrm{iii}}-\mathrm{Rb} 1-\mathrm{O}^{\mathrm{v}}$ & $74.89(14)$ & $\mathrm{O} 3-\mathrm{Na} 1-\mathrm{N} 3^{\mathrm{xii}}$ & $89.85(16)$ \\
\hline $\mathrm{O} 5^{\mathrm{iv}}-\mathrm{Rb} 1-\mathrm{O}^{\mathrm{v}}$ & $142.79(13)$ & $\mathrm{O} 1^{\mathrm{x}}-\mathrm{Na} 1-\mathrm{N} 3^{\mathrm{xii}}$ & $91.21(15)$ \\
\hline $\mathrm{O}^{\mathrm{ii}}-\mathrm{Rb} 1-\mathrm{O}^{\mathrm{v}}$ & $40.29(15)$ & 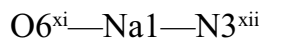 & $24.50(15)$ \\
\hline
\end{tabular}




\begin{tabular}{|c|c|c|c|}
\hline $\mathrm{O} 2-\mathrm{Rb} 1-\mathrm{O} 4^{\mathrm{i}}$ & $68.06(15)$ & 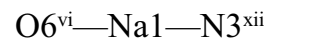 & $101.72(19)$ \\
\hline $\mathrm{O} 2^{\mathrm{i}}-\mathrm{Rb} 1-\mathrm{O} 4^{\mathrm{i}}$ & $98.60(16)$ & $\mathrm{O} 5^{\mathrm{xii}}-\mathrm{Na} 1-\mathrm{N} 3^{\mathrm{xii}}$ & 24.77 (19) \\
\hline $\mathrm{O} 1^{\mathrm{ii}}-\mathrm{Rb} 1-\mathrm{O} 4^{\mathrm{i}}$ & $122.48(14)$ & $\mathrm{O} 5^{\mathrm{vi}}-\mathrm{Na} 1-\mathrm{N} 3^{\mathrm{xii}}$ & $151.0(2)$ \\
\hline 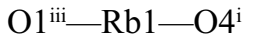 & $69.48(15)$ & $\mathrm{O} 4^{\mathrm{ix}}-\mathrm{Na} 1-\mathrm{N} 3^{\mathrm{xii}}$ & $140.35(18)$ \\
\hline $\mathrm{O}^{\mathrm{iv}}-\mathrm{Rb} 1-\mathrm{O} 4^{\mathrm{i}}$ & $58.44(13)$ & $\mathrm{O} 4^{\mathrm{xiii}}-\mathrm{Na} 1-\mathrm{N} 3^{\mathrm{xii}}$ & $93.42(17)$ \\
\hline $\mathrm{O} 6^{\mathrm{ii}}-\mathrm{Rb} 1-\mathrm{O} 4^{\mathrm{i}}$ & $158.76(14)$ & $\mathrm{O} 3-\mathrm{Na} 1-\mathrm{N} 3^{\mathrm{vi}}$ & $89.85(16)$ \\
\hline $\mathrm{O}^{\mathrm{v}}-\mathrm{Rb} 1-\mathrm{O} 4^{\mathrm{i}}$ & $126.31(13)$ & $\mathrm{O} 1^{\mathrm{x}}-\mathrm{Na} 1-\mathrm{N} 3^{\mathrm{vi}}$ & $91.21(15)$ \\
\hline $\mathrm{O} 2-\mathrm{Rb} 1-\mathrm{O} 4$ & $98.60(16)$ & $\mathrm{O} 6^{\mathrm{xi}}-\mathrm{Na} 1-\mathrm{N} 3^{\mathrm{vi}}$ & $101.72(19)$ \\
\hline $\mathrm{O} 2 \mathrm{i}-\mathrm{Rb} 1-\mathrm{O} 4$ & $68.06(15)$ & $\mathrm{O}^{\mathrm{vi}}-\mathrm{Na} 1-\mathrm{N} 3^{\mathrm{vi}}$ & $24.50(15)$ \\
\hline 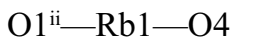 & $69.48(15)$ & $\mathrm{O} 5^{\mathrm{xii}}-\mathrm{Na} 1-\mathrm{N} 3^{\mathrm{vi}}$ & $151.0(2)$ \\
\hline $\mathrm{O}^{\mathrm{iii}-\mathrm{Rb} 1-\mathrm{O} 4}$ & $122.48(14)$ & $\mathrm{O}^{\mathrm{vi}}-\mathrm{Na} 1-\mathrm{N} 3^{\mathrm{vi}}$ & 24.77 (19) \\
\hline $\mathrm{O}^{\mathrm{iv}}-\mathrm{Rb} 1-\mathrm{O} 4$ & $58.44(13)$ & $\mathrm{O} 4^{\mathrm{ix}}-\mathrm{Na} 1-\mathrm{N} 3^{\mathrm{vi}}$ & $93.42(17)$ \\
\hline $\mathrm{O} 6{ }^{\mathrm{ii}}-\mathrm{Rb} 1-\mathrm{O} 4$ & $126.31(13)$ & $\mathrm{O} 4^{\mathrm{xiii}}-\mathrm{Na} 1-\mathrm{N} 3^{\mathrm{vi}}$ & $140.35(18)$ \\
\hline $\mathrm{O}^{\mathrm{v}}-\mathrm{Rb} 1-\mathrm{O} 4$ & $158.76(14)$ & $\mathrm{N} 3^{\mathrm{xii}}-\mathrm{Na} 1-\mathrm{N} 3^{\mathrm{vi}}$ & $126.2(3)$ \\
\hline $\mathrm{O} 4-\mathrm{Rb} 1-\mathrm{O} 4$ & $59.1(2)$ & $\mathrm{O} 3-\mathrm{Na} 1-\mathrm{Rb} 1^{\mathrm{viii}}$ & $130.42(10)$ \\
\hline $\mathrm{O} 2-\mathrm{Rb} 1-\mathrm{O} 2^{\mathrm{ii}}$ & $152.81(8)$ & $\mathrm{O} 1^{\mathrm{x}}-\mathrm{Na} 1-\mathrm{Rb} 1^{\mathrm{viii}}$ & $48.53(8)$ \\
\hline $\mathrm{O} 2^{\mathrm{i}}-\mathrm{Rb} 1-\mathrm{O} 2^{\mathrm{ii}}$ & $112.71(17)$ & $\mathrm{O} 6^{\mathrm{xi}}-\mathrm{Na} 1-\mathrm{Rb} 1^{\mathrm{viii}}$ & $133.97(16)$ \\
\hline $\mathrm{O} 1^{\mathrm{ii}-}-\mathrm{Rb} 1-\mathrm{O} 2^{\mathrm{ii}}$ & $39.33(14)$ & $\mathrm{O}^{\mathrm{vi}}-\mathrm{Na} 1-\mathrm{Rb}^{\mathrm{viii}}$ & $81.38(12)$ \\
\hline 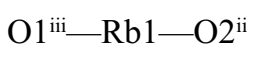 & $94.61(15)$ & $\mathrm{O} 5^{\mathrm{xii}}-\mathrm{Na} 1-\mathrm{Rb} 1^{\mathrm{viii}}$ & $133.96(17)$ \\
\hline $\mathrm{O} 5^{\mathrm{iv}}-\mathrm{Rb} 1-\mathrm{O} 2^{\mathrm{ii}}$ & $78.59(14)$ & $\mathrm{O} 5^{\mathrm{vi}}-\mathrm{Na} 1-\mathrm{Rb} 1^{\mathrm{viii}}$ & $48.89(14)$ \\
\hline $\mathrm{O} 6^{\mathrm{ii}}-\mathrm{Rb} 1-\mathrm{O} 2^{\mathrm{ii}}$ & $64.39(14)$ & $\mathrm{O} 4^{\mathrm{ix}}-\mathrm{Na} 1-\mathrm{Rb} 1^{\mathrm{viii}}$ & $54.16(11)$ \\
\hline $\mathrm{O} 6^{\mathrm{v}}-\mathrm{Rb} 1-\mathrm{O} 2^{\mathrm{ii}}$ & $83.97(13)$ & $\mathrm{O} 4^{\text {xiii }}-\mathrm{Na} 1-\mathrm{Rb} 1^{\text {viii }}$ & $87.31(14)$ \\
\hline $\mathrm{O} 4^{\mathrm{i}}-\mathrm{Rb} 1-\mathrm{O} 2^{\mathrm{ii}}$ & $136.59(15)$ & $\mathrm{N} 3^{\mathrm{xii}}-\mathrm{Na} 1-\mathrm{Rb} 1^{\mathrm{viii}}$ & $139.72(15)$ \\
\hline $\mathrm{O} 4-\mathrm{Rb} 1-\mathrm{O} 2^{\mathrm{ii}}$ & $104.94(15)$ & $\mathrm{N} 3^{\mathrm{vi}}-\mathrm{Na} 1-\mathrm{Rb} 1^{\mathrm{viii}}$ & $63.67(13)$ \\
\hline $\mathrm{O} 2-\mathrm{Rb} 1-\mathrm{O} 2^{\mathrm{v}}$ & $112.71(17)$ & $\mathrm{O} 3-\mathrm{Na} 1-\mathrm{Rb} 1^{\mathrm{xiii}}$ & $130.42(10)$ \\
\hline $\mathrm{O} 2^{\mathrm{i}}-\mathrm{Rb} 1-\mathrm{O} 2^{\mathrm{v}}$ & $152.81(8)$ & $\mathrm{O} 1^{\mathrm{x}}-\mathrm{Na} 1-\mathrm{Rb} 1^{\mathrm{xiii}}$ & $48.53(8)$ \\
\hline 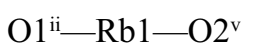 & $94.61(15)$ & 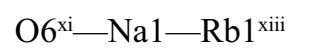 & $81.38(12)$ \\
\hline 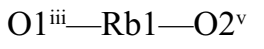 & $39.33(14)$ & $\mathrm{O}^{\mathrm{vi}}-\mathrm{Na} 1-\mathrm{Rb} 1^{\text {xiii }}$ & $133.97(15)$ \\
\hline $\mathrm{O} 5^{\mathrm{iv}}-\mathrm{Rb} 1-\mathrm{O} 2^{\mathrm{v}}$ & $78.59(14)$ & $\mathrm{O} 5^{\mathrm{xii}}-\mathrm{Na} 1-\mathrm{Rb} 1^{\mathrm{xiii}}$ & $48.89(14)$ \\
\hline $\mathrm{O} 6^{\mathrm{ii}-}-\mathrm{Rb} 1-\mathrm{O} 2^{\mathrm{v}}$ & $83.97(13)$ & $\mathrm{O} 5^{\mathrm{vi}}-\mathrm{Na} 1-\mathrm{Rb}^{\mathrm{xiii}}$ & $133.96(17)$ \\
\hline $\mathrm{O} 6^{\mathrm{v}}-\mathrm{Rb} 1-\mathrm{O} 2^{\mathrm{v}}$ & $64.39(14)$ & $\mathrm{O} 4^{\mathrm{ix}}-\mathrm{Na} 1-\mathrm{Rb} 1^{\mathrm{xiii}}$ & $87.31(14)$ \\
\hline $\mathrm{O} 4^{\mathrm{i}}-\mathrm{Rb} 1-\mathrm{O} 2^{\mathrm{v}}$ & $104.94(15)$ & $\mathrm{O} 4^{\mathrm{xii}}-\mathrm{Na} 1-\mathrm{Rb} 1^{\mathrm{xiii}}$ & $54.16(11)$ \\
\hline $\mathrm{O} 4-\mathrm{Rb} 1-\mathrm{O} 2^{\mathrm{v}}$ & $136.59(15)$ & $\mathrm{N} 3^{\mathrm{xii}}-\mathrm{Na} 1-\mathrm{Rb} 1^{\mathrm{xiii}}$ & $63.67(13)$ \\
\hline 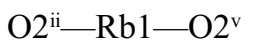 & $56.70(19)$ & $\mathrm{N} 3^{\mathrm{vi}}-\mathrm{Na} 1-\mathrm{Rb} 1^{\mathrm{xiii}}$ & $139.72(15)$ \\
\hline $\mathrm{O} 2-\mathrm{Rb} 1-\mathrm{N} 3^{\mathrm{ii}}$ & $72.37(15)$ & $\mathrm{Rb} 1^{\mathrm{viii}}-\mathrm{Na} 1-\mathrm{Rb} 1^{\mathrm{xiii}}$ & $85.12(9)$ \\
\hline 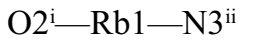 & $72.37(15)$ & $\mathrm{O} 2-\mathrm{N} 1-\mathrm{O} 2^{\mathrm{xiv}}$ & $124.8(8)$ \\
\hline $\mathrm{O} 1^{\mathrm{ii}}-\mathrm{Rb} 1-\mathrm{N} 3^{\mathrm{ii}}$ & $96.71(12)$ & $\mathrm{O} 2-\mathrm{N} 1-\mathrm{O} 1$ & $117.6(4)$ \\
\hline 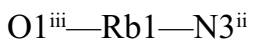 & $96.71(12)$ & $\mathrm{O} 2^{\mathrm{xiv}}-\mathrm{N} 1-\mathrm{O} 1$ & $117.6(4)$ \\
\hline $\mathrm{O} 5^{\mathrm{iv}}-\mathrm{Rb} 1-\mathrm{N} 3^{\mathrm{ii}}$ & $156.66(15)$ & $\mathrm{O} 2-\mathrm{N} 1-\mathrm{Rb}^{\mathrm{xv}}$ & $161.2(5)$ \\
\hline $\mathrm{O} 6^{\mathrm{ii}}-\mathrm{Rb} 1-\mathrm{N} 3^{\mathrm{ii}}$ & $21.85(9)$ & $\mathrm{O} 2^{\mathrm{xiv}}-\mathrm{N} 1-\mathrm{Rb}^{\mathrm{xv}}$ & $67.9(4)$ \\
\hline $\mathrm{O} 6^{\mathrm{v}}-\mathrm{Rb} 1-\mathrm{N} 3^{\mathrm{ii}}$ & $21.85(9)$ & $\mathrm{O} 1-\mathrm{N} 1-\mathrm{Rb}^{\mathrm{xv}}$ & $52.02(16)$ \\
\hline $\mathrm{O} 4{ }^{\mathrm{i}}-\mathrm{Rb} 1-\mathrm{N} 3^{\mathrm{ii}}$ & $139.09(12)$ & $\mathrm{O} 2-\mathrm{N} 1-\mathrm{Rb} 1^{\mathrm{xvi}}$ & $67.9(4)$ \\
\hline $\mathrm{O} 4-\mathrm{Rb} 1-\mathrm{N} 3^{\mathrm{ii}}$ & $139.09(12)$ & $\mathrm{O} 2^{\mathrm{xiv}}-\mathrm{N} 1-\mathrm{Rb} 1^{\mathrm{xvi}}$ & $161.2(5)$ \\
\hline 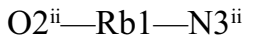 & $80.91(15)$ & $\mathrm{O} 1-\mathrm{N} 1-\mathrm{Rb} 1^{\mathrm{xvi}}$ & $52.02(16)$ \\
\hline $\mathrm{O} 2^{\mathrm{v}}-\mathrm{Rb} 1-\mathrm{N} 3^{\mathrm{ii}}$ & $80.91(15)$ & $\mathrm{Rb} 1^{\mathrm{xv}}-\mathrm{N} 1-\mathrm{Rb} 1^{\mathrm{xvi}}$ & $96.68(18)$ \\
\hline $\mathrm{O}^{\mathrm{vi}}-\mathrm{Rb} 2-\mathrm{O} 3^{\mathrm{vii}}$ & $72.01(12)$ & $\mathrm{N} 1-\mathrm{O} 1-\mathrm{Na} 1^{\mathrm{xvii}}$ & $122.4(5)$ \\
\hline 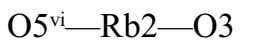 & $72.01(12)$ & $\mathrm{N} 1-\mathrm{O} 1-\mathrm{Rb} 1^{\mathrm{xv}}$ & $108.42(16)$ \\
\hline $\mathrm{O}^{\text {vii }-\mathrm{Rb} 2-\mathrm{O} 3}$ & $124.4(2)$ & $\mathrm{Na} 1^{\mathrm{xvii}}-\mathrm{O} 1-\mathrm{Rb} 1^{\mathrm{xv}}$ & $94.95(12)$ \\
\hline
\end{tabular}




\begin{tabular}{|c|c|c|c|}
\hline $\mathrm{O} 5^{\mathrm{vi}}-\mathrm{Rb} 2-\mathrm{O} 2^{\mathrm{i}}$ & $140.40(15)$ & $\mathrm{N} 1-\mathrm{O} 1-\mathrm{Rb} 1^{\mathrm{xvi}}$ & $108.42(16)$ \\
\hline $\mathrm{O} 3^{\mathrm{vii}}-\mathrm{Rb} 2-\mathrm{O} 2^{\mathrm{i}}$ & $124.77(16)$ & $\mathrm{Na} 1^{\mathrm{xvii}}-\mathrm{O} 1-\mathrm{Rb} 1^{\mathrm{xvi}}$ & $94.95(12)$ \\
\hline $\mathrm{O} 3-\mathrm{Rb} 2-\mathrm{O} 2^{\mathrm{i}}$ & $69.46(17)$ & $\mathrm{Rb} 1^{\mathrm{xv}}-\mathrm{O} 1-\mathrm{Rb} 1^{\mathrm{xvi}}$ & $128.1(2)$ \\
\hline $\mathrm{O} 5^{\mathrm{vi}}-\mathrm{Rb} 2-\mathrm{O} 2$ & $140.40(15)$ & $\mathrm{N} 1-\mathrm{O} 2-\mathrm{Rb} 1$ & $132.5(5)$ \\
\hline 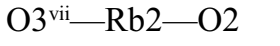 & $69.46(17)$ & $\mathrm{N} 1-\mathrm{O} 2-\mathrm{Rb} 2$ & $135.2(5)$ \\
\hline $\mathrm{O} 3-\mathrm{Rb} 2-\mathrm{O} 2$ & $124.77(16)$ & $\mathrm{Rb} 1-\mathrm{O} 2-\mathrm{Rb} 2$ & $88.08(14)$ \\
\hline $\mathrm{O} 22^{\mathrm{i}}-\mathrm{Rb} 2-\mathrm{O} 2$ & $62.7(2)$ & $\mathrm{N} 1-\mathrm{O} 2-\mathrm{Rb}^{\mathrm{xvi}}$ & $91.9(4)$ \\
\hline $\mathrm{O} 5^{\mathrm{vi}}-\mathrm{Rb} 2-\mathrm{O} 6$ & $125.87(14)$ & $\mathrm{Rb} 1-\mathrm{O} 2-\mathrm{Rb}^{\mathrm{xvi}}$ & $108.5(2)$ \\
\hline $\mathrm{O} 3^{\mathrm{vii}}-\mathrm{Rb} 2-\mathrm{O} 6$ & $97.23(14)$ & $\mathrm{Rb} 2-\mathrm{O} 2-\mathrm{Rb}^{\mathrm{xvi}}$ & $91.11(18)$ \\
\hline $\mathrm{O} 3-\mathrm{Rb} 2-\mathrm{O} 6$ & $138.26(14)$ & $\mathrm{O} 4^{\mathrm{xviii}}-\mathrm{N} 2-\mathrm{O} 4$ & $121.1(9)$ \\
\hline $\mathrm{O} 2 \mathrm{i}-\mathrm{Rb} 2-\mathrm{O} 6$ & $89.98(15)$ & $\mathrm{O} 4^{\mathrm{xviii}}-\mathrm{N} 2-\mathrm{O} 3$ & $119.4(4)$ \\
\hline $\mathrm{O} 2-\mathrm{Rb} 2-\mathrm{O} 6$ & $68.58(15)$ & $\mathrm{O} 4-\mathrm{N} 2-\mathrm{O} 3$ & $119.4(4)$ \\
\hline $\mathrm{O} 5^{\mathrm{vi}}-\mathrm{Rb} 2-\mathrm{O}^{\mathrm{i}}$ & $125.87(14)$ & 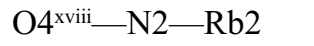 & $159.2(5)$ \\
\hline $\mathrm{O} 3^{\mathrm{vii}}-\mathrm{Rb} 2-\mathrm{O}^{\mathrm{i}}$ & $138.26(13)$ & $\mathrm{O} 4-\mathrm{N} 2-\mathrm{Rb} 2$ & $69.2(4)$ \\
\hline $\mathrm{O} 3-\mathrm{Rb} 2-\mathrm{O}^{\mathrm{i}}$ & $97.23(14)$ & $\mathrm{O} 3-\mathrm{N} 2-\mathrm{Rb} 2$ & $52.92(18)$ \\
\hline $\mathrm{O} 2^{\mathrm{i}}-\mathrm{Rb} 2-\mathrm{O} 6^{\mathrm{i}}$ & $68.58(15)$ & $\mathrm{O} 4^{\mathrm{xviii}}-\mathrm{N} 2-\mathrm{Rb} 2^{\mathrm{xix}}$ & $69.2(4)$ \\
\hline $\mathrm{O} 2-\mathrm{Rb} 2-\mathrm{O} 6^{\mathrm{i}}$ & $89.98(15)$ & $\mathrm{O} 4-\mathrm{N} 2-\mathrm{Rb} 2^{\mathrm{xix}}$ & $159.3(5)$ \\
\hline $\mathrm{O} 6-\mathrm{Rb} 2-\mathrm{O} 6^{\mathrm{i}}$ & $41.04(16)$ & $\mathrm{O} 3-\mathrm{N} 2-\mathrm{Rb} 2^{\mathrm{xix}}$ & $52.92(18)$ \\
\hline $\mathrm{O} 5^{\mathrm{vi}}-\mathrm{Rb} 2-\mathrm{O} 4^{\mathrm{viii}}$ & $57.95(13)$ & $\mathrm{Rb} 2-\mathrm{N} 2-\mathrm{Rb} 2^{\mathrm{xix}}$ & $95.54(18)$ \\
\hline $\mathrm{O} 3^{\text {vii }}-\mathrm{Rb} 2-\mathrm{O} 4^{\text {viii }}$ & $69.19(16)$ & $\mathrm{N} 2-\mathrm{O} 3-\mathrm{Na} 1$ & $127.6(5)$ \\
\hline $\mathrm{O} 3-\mathrm{Rb} 2-\mathrm{O} 4^{\text {viii }}$ & $120.69(15)$ & $\mathrm{N} 2-\mathrm{O} 3-\mathrm{Rb} 2$ & $107.6(2)$ \\
\hline $\mathrm{O} 2^{\mathrm{i}}-\mathrm{Rb} 2-\mathrm{O} 4^{\text {viii }}$ & $156.66(17)$ & $\mathrm{Na} 1-\mathrm{O} 3-\mathrm{Rb} 2$ & $95.57(15)$ \\
\hline $\mathrm{O} 2-\mathrm{Rb} 2-\mathrm{O} 4^{\text {viii }}$ & $114.13(14)$ & $\mathrm{N} 2-\mathrm{O} 3-\mathrm{Rb} 2^{\mathrm{xix}}$ & $107.6(2)$ \\
\hline $\mathrm{O} 6-\mathrm{Rb} 2-\mathrm{O} 4^{\text {viii }}$ & $68.52(13)$ & $\mathrm{Na} 1-\mathrm{O} 3-\mathrm{Rb}^{\mathrm{xix}}$ & $95.57(15)$ \\
\hline $\mathrm{O} 6^{\mathrm{i}}-\mathrm{Rb} 2-\mathrm{O} 4^{\mathrm{vii}}$ & $88.67(15)$ & $\mathrm{Rb} 2-\mathrm{O} 3-\mathrm{Rb} 2^{\mathrm{xix}}$ & $124.4(2)$ \\
\hline $\mathrm{O} 5^{\mathrm{vi}}-\mathrm{Rb} 2-\mathrm{O} 4^{\mathrm{ix}}$ & $57.95(13)$ & $\mathrm{N} 2-\mathrm{O} 4-\mathrm{Na} 1^{\mathrm{xii}}$ & $96.0(5)$ \\
\hline $\mathrm{O} 3^{\mathrm{vii}}-\mathrm{Rb} 2-\mathrm{O} 4^{\mathrm{ix}}$ & $120.69(15)$ & $\mathrm{N} 2-\mathrm{O} 4-\mathrm{Rb} 1$ & $127.6(4)$ \\
\hline $\mathrm{O} 3-\mathrm{Rb} 2-\mathrm{O} 4^{\mathrm{ix}}$ & $69.19(16)$ & $\mathrm{Na} 1^{\mathrm{xii}}-\mathrm{O} 4-\mathrm{Rb} 1$ & $82.92(15)$ \\
\hline $\mathrm{O} 2^{\mathrm{i}}-\mathrm{Rb} 2-\mathrm{O} 4^{\mathrm{ix}}$ & $114.13(14)$ & $\mathrm{N} 2-\mathrm{O} 4-\mathrm{Rb}^{\mathrm{vi}}$ & $125.5(4)$ \\
\hline $\mathrm{O} 2-\mathrm{Rb} 2-\mathrm{O} 4^{\mathrm{ix}}$ & $156.66(17)$ & $\mathrm{Na} 1^{\mathrm{xii}}-\mathrm{O} 4-\mathrm{Rb} 2^{\mathrm{vi}}$ & $83.53(17)$ \\
\hline $\mathrm{O} 6-\mathrm{Rb} 2-\mathrm{O} 4^{\mathrm{ix}}$ & $88.67(15)$ & $\mathrm{Rb} 1-\mathrm{O} 4-\mathrm{Rb} 2^{\mathrm{vi}}$ & $106.46(15)$ \\
\hline $\mathrm{O} 6^{\mathrm{i}}-\mathrm{Rb} 2-\mathrm{O} 4^{\mathrm{ix}}$ & $68.52(13)$ & $\mathrm{N} 2-\mathrm{O} 4-\mathrm{Rb} 2$ & $90.7(4)$ \\
\hline $\mathrm{O} 4^{\mathrm{viii}}-\mathrm{Rb} 2-\mathrm{O} 4^{\mathrm{ix}}$ & $58.6(2)$ & 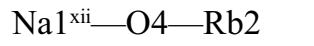 & $160.12(19)$ \\
\hline $\mathrm{O} 5^{\mathrm{vi}}-\mathrm{Rb} 2-\mathrm{N} 3$ & $120.62(15)$ & $\mathrm{Rb} 1-\mathrm{O} 4-\mathrm{Rb} 2$ & $78.21(13)$ \\
\hline $\mathrm{O} 3^{\mathrm{vii}-\mathrm{Rb} 2-\mathrm{N} 3}$ & $117.24(11)$ & $\mathrm{Rb} 2 \mathrm{vi}-\mathrm{O} 4-\mathrm{Rb} 2$ & $107.71(17)$ \\
\hline $\mathrm{O} 3-\mathrm{Rb} 2-\mathrm{N} 3$ & $117.24(11)$ & $\mathrm{O} 6 \mathrm{i}-\mathrm{N} 3-\mathrm{O} 6$ & $119.5(7)$ \\
\hline $\mathrm{O} 22^{\mathrm{i}}-\mathrm{Rb} 2-\mathrm{N} 3$ & $85.64(15)$ & $\mathrm{O} 66^{\mathrm{i}}-\mathrm{N} 3-\mathrm{O} 5$ & $120.3(3)$ \\
\hline $\mathrm{O} 2-\mathrm{Rb} 2-\mathrm{N} 3$ & $85.64(15)$ & $\mathrm{O} 6-\mathrm{N} 3-\mathrm{O} 5$ & $120.3(3)$ \\
\hline $\mathrm{O} 6-\mathrm{Rb} 2-\mathrm{N} 3$ & $22.00(9)$ & $\mathrm{O}^{2}-\mathrm{N} 3-\mathrm{Na} 1^{\text {viii }}$ & $176.6(4)$ \\
\hline $\mathrm{O} 6{ }^{\mathrm{i}}-\mathrm{Rb} 2-\mathrm{N} 3$ & $22.00(9)$ & $\mathrm{O} 6-\mathrm{N} 3-\mathrm{Na} 1^{\mathrm{viii}}$ & $57.2(3)$ \\
\hline 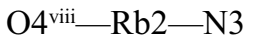 & $71.02(15)$ & $\mathrm{O} 5-\mathrm{N} 3-\mathrm{Na} 1^{\mathrm{viii}}$ & $63.11(13)$ \\
\hline $\mathrm{O} 4^{\mathrm{ix}}-\mathrm{Rb} 2-\mathrm{N} 3$ & $71.02(14)$ & $\mathrm{O} 6^{\mathrm{i}}-\mathrm{N} 3-\mathrm{Na} 1^{\mathrm{xiii}}$ & $57.2(3)$ \\
\hline $\mathrm{O} 5^{\mathrm{vi}}-\mathrm{Rb} 2-\mathrm{O} 4^{\mathrm{i}}$ & $78.96(13)$ & $\mathrm{O} 6-\mathrm{N} 3-\mathrm{Na} 1^{\mathrm{xiii}}$ & $176.6(4)$ \\
\hline $\mathrm{O} 3^{\mathrm{vii}}-\mathrm{Rb} 2-\mathrm{O} 4^{\mathrm{i}}$ & $39.02(14)$ & $\mathrm{O} 5-\mathrm{N} 3-\mathrm{Na} 1^{\mathrm{xiii}}$ & $63.11(13)$ \\
\hline $\mathrm{O} 3-\mathrm{Rb} 2-\mathrm{O} 4^{\mathrm{i}}$ & $93.33(15)$ & $\mathrm{Na} 1^{\mathrm{vii} \_\mathrm{N}} 3-\mathrm{Na} 1^{\mathrm{xiii}}$ & $126.2(3)$ \\
\hline $\mathrm{O} 2^{\mathrm{i}}-\mathrm{Rb} 2-\mathrm{O} 4^{\mathrm{i}}$ & $94.40(14)$ & $\mathrm{O} 6{ }^{\mathrm{i}}-\mathrm{N} 3-\mathrm{Rb} 2$ & $67.3(4)$ \\
\hline $\mathrm{O} 2-\mathrm{Rb} 2-\mathrm{O} 4^{\mathrm{i}}$ & $65.50(16)$ & $\mathrm{O} 6-\mathrm{N} 3-\mathrm{Rb} 2$ & $67.3(4)$ \\
\hline $\mathrm{O} 6-\mathrm{Rb} 2-\mathrm{O} 4^{\mathrm{i}}$ & $125.14(12)$ & $\mathrm{O} 5-\mathrm{N} 3-\mathrm{Rb} 2$ & $140.2(4)$ \\
\hline
\end{tabular}




\begin{tabular}{|c|c|c|c|}
\hline $\mathrm{O} 6-\mathrm{Rb} 2-\mathrm{O}^{\mathrm{i}}$ & $155.01(12)$ & $\mathrm{Na} 1^{\mathrm{viii}}-\mathrm{N} 3-\mathrm{Rb} 2$ & $110.63(14)$ \\
\hline 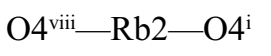 & $105.26(15)$ & 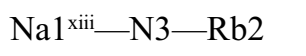 & $110.63(14)$ \\
\hline $\mathrm{O} 4^{\mathrm{ix}}-\mathrm{Rb} 2-\mathrm{O} 4^{\mathrm{i}}$ & $136.46(9)$ & $\mathrm{O}^{\mathrm{i}}-\mathrm{N} 3-\mathrm{Rb} 1^{\mathrm{xvi}}$ & $68.9(3)$ \\
\hline $\mathrm{N} 3-\mathrm{Rb} 2-\mathrm{O} 4^{\mathrm{i}}$ & $146.94(11)$ & $\mathrm{O} 6-\mathrm{N} 3-\mathrm{Rb} 1^{\mathrm{xvi}}$ & $68.9(3)$ \\
\hline $\mathrm{O} 5{ }^{\mathrm{vi}}-\mathrm{Rb} 2-\mathrm{O} 4$ & $78.96(13)$ & $\mathrm{O} 5-\mathrm{N} 3-\mathrm{Rb} 1^{\mathrm{xvi}}$ & $135.1(5)$ \\
\hline $\mathrm{O}^{\mathrm{vii}}-\mathrm{Rb} 2-\mathrm{O} 4$ & $93.33(15)$ & 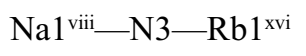 & $108.37(13)$ \\
\hline $\mathrm{O} 3-\mathrm{Rb} 2-\mathrm{O} 4$ & $39.02(14)$ & $\mathrm{Na} 1^{\mathrm{xiii}}-\mathrm{N} 3-\mathrm{Rb} 1^{\mathrm{xvi}}$ & $108.37(13)$ \\
\hline $\mathrm{O} 2-\mathrm{Rb} 2-\mathrm{O} 4$ & $65.50(16)$ & $\mathrm{Rb} 2-\mathrm{N} 3-\mathrm{Rb}^{\mathrm{xvi}}$ & $84.67(16)$ \\
\hline $\mathrm{O} 2-\mathrm{Rb} 2-\mathrm{O} 4$ & $94.40(14)$ & $\mathrm{N} 3-\mathrm{O} 5-\mathrm{Na} 1^{\text {viii }}$ & $92.12(15)$ \\
\hline $\mathrm{O} 6-\mathrm{Rb} 2-\mathrm{O} 4$ & $155.01(12)$ & $\mathrm{N} 3-\mathrm{O} 5-\mathrm{Na} 1^{\text {xiii }}$ & $92.12(15)$ \\
\hline $\mathrm{O} 6-\mathrm{Rb} 2-\mathrm{O} 4$ & $125.14(12)$ & 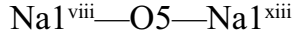 & $175.6(3)$ \\
\hline 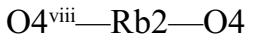 & $136.46(9)$ & $\mathrm{N} 3-\mathrm{O} 5-\mathrm{Rb} 1^{\mathrm{xx}}$ & $119.1(4)$ \\
\hline $\mathrm{O} 4^{\mathrm{ix}}-\mathrm{Rb} 2-\mathrm{O} 4$ & $105.26(14)$ & $\mathrm{Na} 1^{\mathrm{viii}}-\mathrm{O} 5-\mathrm{Rb} 1^{\mathrm{xx}}$ & $88.53(15)$ \\
\hline $\mathrm{N} 3-\mathrm{Rb} 2-\mathrm{O} 4$ & $146.94(11)$ & $\mathrm{Na} 1^{\mathrm{xiii}}-\mathrm{O} 5-\mathrm{Rb} 1^{\mathrm{xx}}$ & $88.53(15)$ \\
\hline $\mathrm{O} 4 \mathrm{i}-\mathrm{Rb} 2-\mathrm{O} 4$ & $56.31(18)$ & $\mathrm{N} 3-\mathrm{O} 5-\mathrm{Rb} 2^{\text {viii }}$ & $120.2(5)$ \\
\hline $\mathrm{O} 3-\mathrm{Na} 1-\mathrm{O}^{\mathrm{x}}$ & $177.7(3)$ & $\mathrm{Na} 1^{\text {viii_-O } 5-\mathrm{Rb} 2^{\text {viii }}}$ & 89.37 (14) \\
\hline $\mathrm{O} 3-\mathrm{Na} 1-\mathrm{O}^{\mathrm{xi}}$ & $90.1(2)$ & $\mathrm{Na} 1^{\text {xiii }}-\mathrm{O} 5-\mathrm{Rb} 2^{\text {viii }}$ & $89.37(14)$ \\
\hline $\mathrm{O} 1^{\mathrm{x}}-\mathrm{Na} 1-\mathrm{O}^{\mathrm{xi}}$ & $91.76(19)$ & $\mathrm{Rb} 1^{\mathrm{xx}}-\mathrm{O} 5-\mathrm{Rb} 2^{\mathrm{viii}}$ & $120.7(2)$ \\
\hline $\mathrm{O} 3-\mathrm{Na} 1-\mathrm{O}^{\mathrm{vi}}$ & $90.1(2)$ & $\mathrm{N} 3-\mathrm{O} 6-\mathrm{Na} 1^{\mathrm{vii}}$ & $98.3(4)$ \\
\hline $\mathrm{O}^{\mathrm{x}}-\mathrm{Na} 1-\mathrm{O}{ }^{\mathrm{vi}}$ & $91.76(19)$ & $\mathrm{N} 3-\mathrm{O} 6-\mathrm{Rb} 2$ & $90.7(4)$ \\
\hline $\mathrm{O} 6^{\mathrm{xi}}-\mathrm{Na} 1-\mathrm{O}^{\mathrm{vi}}$ & $77.2(2)$ & $\mathrm{Na} 1^{\mathrm{viii}-\mathrm{O} 6-\mathrm{Rb} 2}$ & $135.6(2)$ \\
\hline $\mathrm{O} 3-\mathrm{Na} 1-\mathrm{O} 5^{\mathrm{xii}}$ & $89.52(14)$ & $\mathrm{N} 3-\mathrm{O} 6-\mathrm{Rb} 1^{\mathrm{xvi}}$ & $89.2(4)$ \\
\hline $\mathrm{O} 1^{\mathrm{x}}-\mathrm{Na} 1-\mathrm{O}^{\mathrm{xii}}$ & $90.56(14)$ & $\mathrm{Na} 1^{\mathrm{viii}}-\mathrm{O} 6-\mathrm{Rb}^{\mathrm{xvi}}$ & $130.22(19)$ \\
\hline $\mathrm{O}^{\mathrm{xi}}-\mathrm{Na} 1-\mathrm{O} 5^{\mathrm{xii}}$ & $49.27(16)$ & $\mathrm{Rb} 2-\mathrm{O} 6-\mathrm{Rb}^{\mathrm{xvi}}$ & $93.04(12)$ \\
\hline $\mathrm{O} 6^{\mathrm{vi}}-\mathrm{Na} 1-\mathrm{O} 5^{\mathrm{xii}}$ & $126.5(2)$ & & \\
\hline
\end{tabular}

Symmetry codes: (i) $-x+1, y, z$; (ii) $-x+1,-y, z-1 / 2$; (iii) $-x+2,-y, z-1 / 2$; (iv) $x, y, z-1$; (v) $x,-y, z-1 / 2$; (vi) $-x+1,-y+1, z-1 / 2$; (vii) $x+1, y, z$; (viii) $-x+1,-y+1, z+1 / 2$; (ix) $x,-y+1, z+1 / 2$; (x) $x-1, y+1, z$; (xi) $x-1,-y+1, z-1 / 2$; (xii) $-x,-y+1, z-1 / 2$; (xiii) $-x,-y+1, z+1 / 2$; (xiv) $-x+2, y, z$; (xv) $-x+2,-y$, $z+1 / 2$; (xvi) $-x+1,-y, z+1 / 2$; (xvii) $x+1, y-1, z$; (xviii) $-x, y, z$; (xix) $x-1, y, z$; (xx) $x, y, z+1$. 